\title{
Functional dynamic bracing and functional rehabilitation for Achilles tendon ruptures: a case series
}

\author{
Philippa Dolphin ${ }^{1}$, Kelly Bainbridge ${ }^{1}$, Paul Mackenney ${ }^{2}$, John Dixon ${ }^{3 \S}$ \\ ${ }^{1}$ Department of Rehabilitation, James Cook University Hospital, Middlesbrough, UK \\ ${ }^{2}$ Department of Orthopaedics, James Cook University Hospital, Middlesbrough, UK \\ ${ }^{3}$ Health and Social Care Institute, Teesside University, Middlesbrough, UK
}

§Corresponding author address:

Prof John Dixon PhD, Health and Social Care Institute, Teesside University, Middlesbrough, TS1 3BA, UK.

Email address: john.dixon@,tees.ac.uk

Telephone: +44 (0)1642 384125, Fax +44 (0)1642 342983

2545 words

Email addresses:

PD: Philippa.Dolphin@stees.nhs.uk

KB: Kelly.Bainbridge@stees.nhs.uk

PM:paul.mackenney@stees.nhs.uk

JD: john.dixon@tees.ac.uk 


\section{Abstract}

\section{Background}

There has been considerable debate surrounding both the surgical and conservative management of Achilles tendon rupture. There is now a trend towards conservative management however there is no consensus on the best method for this. The aim of this study was to evaluate the clinical and functional outcomes of the conservative management of Achilles tendon rupture using functional dynamic bracing within a functional rehabilitation programme.

\section{Methods}

This was a case-series (an uncontrolled before-after) study, and 25 participants with acute Achilles tendon rupture were recruited. The intervention was a conservative management programme with functional dynamic bracing and functional rehabilitation. The primary outcome measure was re-rupture. Secondary outcome measures were the change in EQ-5D$5 \mathrm{~L}$ VAS and index scores between commencement of management and 6 months follow up, and the Achilles Tendon Total Rupture Score (ATRS) at 6 months follow up.

\section{Results}

No re-ruptures occurred in those participants who complied with the programme $(0 / 21)$. There were statistically significant positive changes in the EQ-5D-5L VAS with a mean (95\% $\mathrm{Cl}$ ) difference pre-post treatment of 12.9 (4.2 to 21.6), $\mathrm{p}=0.006$. The EQ-5D-5L index scores also significantly improved (Wilcoxon signed-rank test $p<0.001$ ). The mean (SD) ATRS score at 6 months follow up was 76.9 (19.9).

\section{Conclusions}


The results of this study show management of Achilles tendon rupture using functional dynamic bracing within a functional rehabilitation programme produces good clinical outcomes. This study strengthens the evidence base for this conservative intervention in Achilles tendon ruptures.

Keywords: Achilles tendon; rupture; physiotherapy; rehabilitation; treatment; 


\section{Introduction}

The Achilles tendon is the thickest and strongest tendon in the human body and is also the most frequently ruptured. There has been considerable debate around whether Achilles tendon rupture is most effectively managed by surgical intervention or conservative treatment [1]. Traditionally, conservative management consisted of plaster cast immobilisation for $10-12$ weeks. Whilst surgery carries risk of complications, it has been observed that conservative management in traditional plaster cast immobilisation has a higher risk of re-rupture than surgical management [2]. However, where functional rehabilitation is used as a part of conservative management, the re-rupture rate has been reported as similar to that of surgical management [3]. This is an important area and a decline in the rate of surgery for Achilles tendon ruptures has been reported [4].

Functional rehabilitation has been described in a number of papers $[5,6]$ and generally refers to an orthosis that permits weight bearing, but does not necessarily allow for movement at the ankle. Functional bracing is described within the literature but has not been clearly defined. This phrase usually applies to a weight bearing orthosis but does not always indicate whether the device permits movement at the ankle. Functional Bracing may include dynamic bracing, rigid dorsal split, removable cast boot or semi-rigid wrap [6]. There are a number of studies investigating functional rehabilitation following surgery for Achilles tendon rupture [6]. But there is little published research regarding the effectiveness of conservative functional rehabilitation for Achilles tendon ruptures using functional dynamic bracing such as the VACOped boot (OPED UK Ltd, Devizes, UK). Neumayer et al. [7] reported low rates of re-rupture (9\%) using this intervention, but did not collect any validated patient 
reported outcome measures. Hutchison et al. [8] reported a re-rupture rate of $1 \%$ in a descriptive case series study of a dedicated conservative management programme incorporating functional dynamic bracing.

In light of this scarcity of published evidence, the aim of this study was to evaluate the clinical and functional outcomes of conservative rehabilitation of Achilles tendon ruptures using functional dynamic bracing within a functional rehabilitation programme. This study used the VACOped, which has a rigid shell but may be dynamised to allow increasing range of movement at the ankle through the rehabilitation process. The primary outcome measure was re-rupture. Secondary outcome measures were EQ-5D-5L VAS and index scores, and the Achilles Tendon Total Rupture Score (ATRS) at 6 months follow up.

\section{Methods}

\section{Design}

We conducted a case-series (prospective uncontrolled longitudinal) study. Clinical outcome measures were collected before and after a 6 months treatment program using functional rehabilitation.

\section{Participants}

We considered for inclusion into the study all patients that were referred to the physiotherapist led ankle soft tissue injury clinic from A\&E with suspected first time acute Achilles tendon rupture. Inclusion criteria were: men and women, 18 years of age and over, with the first episode of an acute Achilles tendon rupture; ability to read and comprehend 
written and spoken English. Exclusion Criteria were: delayed presentation, where a patient was not immobilised in equinus within 48 hours; any doubt of ability of the participant to give informed consent; inability to understand written and spoken English. As an exploratory study, the recruitment period was set for six months (with six months followup), and no a priori sample size estimates were made. The study was approved by the East Midlands-Northants Research Ethics Committee (Ref 14/EM/1040). All participants gave informed consent.

\section{Procedure}

Rupture was diagnosed using the subjective history, palpation and the Thompson Test (calf squeeze test) [9]. This was done by the lead author.

Patients were deemed appropriate for conservative management if the Achilles tendon defect approximated when the ankle was placed into full equinus. In cases where palpation did not clearly demonstrate that the defect would fully approximate in equinus, confirmation would have been sought via ultrasound scan. This was not deemed necessary in any of the participants. Patients in whom there was no approximation of the tendon ends were referred for surgical repair and were excluded from the study. Once the decision to manage conservatively had been made, the patient was fitted with a VACOped boot (OPED UK Ltd, Devizes, UK), shown in Figure 1, set at 30 degrees of plantar flexion. The patient was instructed to weight bear as tolerated. At this appointment patients were invited to participate in the study. Participants' data were pseudo-anonymised at the time of inclusion. All patients were assessed for risk of venous thromboembolism (VTE), and patients deemed to be high risk were managed with prophylaxis. 
Insert Figure 1 here.

All patients were seen by the same Extended Scope Physiotherapy Practitioner at each review appointment. They were reviewed at 4, 6, 8 and 10 weeks post rupture. At each review appointment, the Achilles was examined in prone and the Achilles tendon palpated for presence of defect. Adjustments were made at each appointment as detailed in Table 1. At 10 weeks post-rupture the boot was removed and the patient fitted with a standard heel raise which was gradually reduced in size over 5 weeks. (MAG shoe services: $15 \mathrm{~mm} \mathrm{x}$ $130 \mathrm{~mm}$ male; $15 \mathrm{~mm} \times 110 \mathrm{~mm}$ female) and a lower limb strengthening program was begun in physiotherapy. At this point the physiotherapy was tailored to each individual's needs in terms of general health and fitness, lifestyle demands and goals. The common principles in rehabilitation were to restore proprioception, strength and control. Stretching into dorsiflexion was avoided in all participants until 16 weeks and when relevant participants returned to light jogging at 16 weeks following rupture. Patients who did not live close to the teaching hospital were given an option to access a physiotherapy department closer to home once the boot had been removed.

Insert Table 1 here.

\section{Outcome measures}

All patients were followed up in the ankle soft tissue clinic at 6 months post injury where outcome measures were collected. The primary outcome measure of the study, re-rupture, was assessed by palpation at six months. Participants completed an EQ-5D-5L questionnaire at first assessment and at the 6-month assessment. This is a recommended generic patient 
reported outcome measure of health status $[10,11]$. It has two parts, the descriptive system with five dimensions, and the visual analogue scale (VAS) for health in which patients assess their health on that day. Both parts were evaluated for each patient. Firstly the VAS score was extracted. Secondly the EQ-5D index was calculated, from the descriptive system data using the UK value set [12], giving a single index score where 1 equates to 'full health' and 0 equates to death. Participants also completed the Achilles Tendon Total Rupture Score (ATRS) questionnaire, a condition-specific patient reported outcome measure which assesses symptoms and physical activity. This has been shown to be a valid and reliable outcome measure specifically for Achilles tendon ruptures $[13,14]$ The ATRS is a ten item questionnaire, the patient is asked to score each item on a scale of 0 to 10,10 being most positive. The maximum score is 100 . This was completed at the 6 months assessment only. It was not completed at baseline as the patients were already immobilised when seen in clinic, and therefore may have not been able to reliably or objectively report symptoms. Any complications were documented from the patient database and clinical assessment.

\section{Statistical analysis}

Descriptive analysis was used for the re-rupture, demographic, ATRS, and complications data. Inferential analyses were performed to assess changes in the EQ-5D-5L VAS and index between baseline and 6 months. The data were tested for normal distribution using the Shapiro Wilk test. As the EQ-5D-5L VAS data were found to have a normal distribution $(p>0.05)$ the analysis was carried out with a paired $t$ test. As the EQ-5D-5L index data was found to breach the assumptions of normality $(p<0.05)$ the analysis was carried out with a Wilcoxon signed-rank test. Inferential tests were two-tailed with alpha set at $p=0.05$. Data were analysed with SPSS version 22.0 (SPSS Inc, Chicago, IL, 60606, USA). 


\section{Results}

The study recruited for a six month period. A total of 25 patients were recruited into the study, and 4 were lost to follow-up. Of those 4, 1 withdrew as they sought opinion elsewhere, 2 were excluded due other injuries sustained at the time of Achilles rupture, 1 was excluded due to non-compliance with instructions to wear the boot at all times and early removal of boot (subsequently requiring Achilles tendon repair at 4 weeks post rupture). The remaining 21 participants completed the study and their descriptive characteristics are shown in Table 2.

Insert Table 2 here.

\section{Primary outcome: re-rupture}

There were no re-ruptures in those who complied with the programme, and so the rate of re-rupture during the 6 month study was $0 \%(0 / 21)$.

\section{EQ-5D-5L}

Data were analysed for 17 participants due to incomplete data. The mean (SD) EQ-5D-5L VAS score was 72.9 (17.1) pre-treatment, and this improved to 85.8 (9.8) post-treatment, as shown in Figure 2 . The paired t test showed the mean $(95 \% \mathrm{Cl})$ difference pre-post treatment was 12.9 (4.2 to 21.6), $p=0.006$. The median (IQR) EQ5D-5L index value was $0.673(0.09)$ pre-treatment, and this improved to $1.00(0.24)$ post-treatment. The Wilcoxon signed-rank test showed that this improvement was statistically significant, $p<0.001$. 
Insert Figure 2 here.

\section{ATRS}

At 6 months the mean ATRS score was 76.9 (19.9), range 21-97 $(n=19)$.

\section{Study complications}

There were no incidences of VTE or skin breakdown observed. No patients scored highly in VTE risk assessment, so in no cases was prophylaxis required.

\section{Discussion}

The aim of this study was to evaluate the clinical and functional outcomes of conservative rehabilitation of Achilles tendon ruptures using functional dynamic bracing within a functional rehabilitation programme. The results showed positive findings; no re-ruptures or complications were observed in participants who completed the programme, there were significant improvements in EQ-5D-5L results after 6 months, and the ATRS scores were positive. To the authors' knowledge, this is the first published study to quantify this intervention using the EQ-5D-5L outcome measure.

The results of this study compare well with previous reports of conservative functional rehabilitation interventions. The zero re-rupture rate in those who complied with the programme and completed the study $(0 / 21)$ is similar to the results from Hutchison et al. [8], who reported $1.1 \%(3 / 273)$. Our rate is lower than other studies such as Neumayer et al. [7] who reported 9\%, with 5 full re-ruptures and 2 partial ones, in 57 patients, and Weber et al. [15] who reported a re-rupture rate of $17 \%(4 / 23)$. It is notable that the current study 
reports a similar re-rupture rate to Hutchison et al. [8] who attributed their low rate to various factors including a detailed ultrasonographic examination, a dedicated physiotherapist led Achilles tendon rupture clinic and use of a physiotherapy protocol. The current study was also based on a dedicated physiotherapist led Achilles tendon rupture clinic, however it differs from the Hutchison regimen as it did not use ultrasound scan for assessment on all patients.

The current study observed an improvement in the mean EQ-5D-5L VAS from 72.9 to 85.8. This mean change of 12.9 units equates to a standardised effect size of approximately 0.75 , indicating a medium sized effect, close to the 0.8 value classed as large [16]. The EQ-5D-5L has rarely been used in research studies of Achilles tendon rehabilitation, or foot and ankle rehabilitation generally. In a study of 74 patients two years after ankle fracture, Lash et al. [17] reported average EQ-5D VAS values ranging from 70 to 86 , depending on fracture type. These data seem comparable to values in the current study. The significant improvement in the EQ-5D-5L index also appears clinically relevant, and compares well with a recent validity study which observed mean EQ-5D-3L index scores of 0.90 six months after conservative treatment of Achilles ruptures [14]. Importantly, the EQ-5D-5L data from the current study may also be useful in estimating sample sizes for studies of economic evaluation in the field.

The ATRS results of the current study at 6 months post-rupture look similar to other studies. Kearney et al. [14] reported mean (SD) ATRS values of 65 (23) at six months post injury in non-operative rehabilitation. Of interest, the current study found the mean ATRS score to be 76.9 at 6 months in comparison to the value of 67.8 reported in the study by Hutchison et al. [8]. The physiotherapy protocol also differed between the current study and that of 
Hutchison et al. [8], with the current study advocating introduction of jogging from 4 months post rupture rather than 5 months as described by Hutchison. As previous research [13] has estimated a 10 point difference in ATRS scores to be clinically significant, the difference between the outcome scores of the two studies could perhaps be attributed to the difference in rehabilitation protocols with the earlier introduction of jogging in the current study. Overall, the results of our study, although limited by small sample size, add to the understanding of this type of intervention with clinical data from validated outcome measures, both generic and condition-specific.

The current findings have relevance for clinical practice as they support conservative management of Achilles tendon rupture using functional dynamic bracing. The results indicate that this treatment regimen has clear clinical benefits for patients. In addition, with no re-ruptures in those who complied with the programme, the findings indicate that the potential re-rupture risk in conservative treatment can be overcome with this intervention. This is a role which has traditionally been carried out by orthopaedic surgeons; this study demonstrates that it can be performed effectively by physiotherapists. In addition, it should be noted that this occurred without the necessity for ultrasound scanning. So this study demonstrates the potential benefits of a functional rehabilitation programme for use with conservatively managed Achilles tendon ruptures.

There are limitations to the current study. The key limitation was the absence of a control group, as this was a case-series study not a randomised controlled trial. In addition we did not use random sampling which limits the generalisability of our findings. However it has been argued that observational studies provide important information, for example when it 
is unethical to randomise $[18,19]$. Patients were only followed up until 6 months post injury and it can therefore not be determined that these patients did not go onto rupture at a later stage. A further limitation would be the small sample size of only 21 participants completing the study from an initial 25 . Nevertheless the study recruited for a six month period and this sample is representative of the population in our setting.

Future studies should use a control group, possibly in multi-centre randomised controlled trials, to better assess and perhaps refine the effectiveness of functional dynamic bracing and functional rehabilitation in patients with Achilles tendon ruptures.

\section{Conclusions}

The results of this case-series study show that conservative management of Achilles tendon ruptures using functional dynamic bracing within a functional rehabilitation programme produced zero re-ruptures and good clinical outcomes.

\section{Acknowledgments}

We would like to thank all the patients who kindly volunteered to take part in this study, and Scott Murray for advice on orthotic management. A small grant from the Council for Allied Health Professions Research was received to support the project. PD received modest educational support from OPED UK Ltd (Medical Devices) to attend a conference. 


\section{References}

1. Wilkins R, Bisson $\sqcup$. Operative versus nonoperative management of acute Achilles tendon ruptures: A quantitative systematic review of randomized controlled trials. Am J Sports Med. 2012; 40: 2154-60.

2. Khan RJK, Fick D, Keogh A, Crawford J, Brammar T, Parker M. Treatment of Achilles tendon ruptures. A meta-analysis of randomised controlled trials. J Bone Joint Surg Am. $2005 ; 87: 2202-10$.

3. Soroceanu A, Sidhwa F, Aarabi S, Kaufman A, Glazebrook M. Surgical Versus Nonsurgical Treatment of Acute Achilles Tendon Rupture. J Bone Joint Surg Am. 2012; 94: 2136-43.

4. Mattila VM, Huttenen TT, Haapasalo H, Sillanpaa P, Malmivaara A, Pihlajamaki. Declining incidence of surgery for Achilles tendon rupture follows publication of major RCTs: evidence-influenced change evident using the Finnish registry study. Br J Sports Med. 2015; 49:1084-86

5. Kearney R, McGuiness K, Achten J, Costa M. A systematic review of the early rehabilitation methods following a rupture of the Achilles tendon. Physiother. 2012; 98: 2432 
6 McCormack R, Bovard J. Early functional rehabilitation or cast immobilisation for the postoperative management of acute Achilles tendon rupture? A systematic review and meta-analysis of randomised controlled trials. Br J Sports Med. 2015; 49:1329-35.

7. Neumayer F, Mouhsine E, Arlettaz Y, Gremion G, Wettstein M, Crevoisier X. A new conservative-dynamic treatment for the acute ruptured Achilles tendon. Arch Orthop Trauma Surg. 2010; 130: 363-8.

8. Hutchison AM, Topliss C, Beard D, Evans RM, Williams P. The treatment of a rupture of the Achilles tendon using a dedicated management programme. Bone and Joint Journal. 2015; 97-B: 510-15

9. Chiodo CP, Glazebrook M, Bluman EM, Cohen B, Femino J, Giza E, et al. Diagnosis and treatment of acute Achilles tendon rupture. J Am Acad Orthop Surg. 2010; 18:503-510.

10. Janssen MF, Pickard AS, Golicki D, Gudex C, Niewada M, Scalone L, et al. Measurement properties of the EQ-5D-5L compared to the EQ-5D-3L across eight patient groups: a multicountry study. Qual Life Res. 2012; doi: 10.1007/s11136-012-0322-4.

11. Rabin R, de Charro F. EQ-5D: a measure of health status from the EuroQol Group. Ann Med. 2001; 33:337-43. 
12. Chartered Society of Physiotherapy, 2011. EQ-5D-5L - Calculator and explanation.

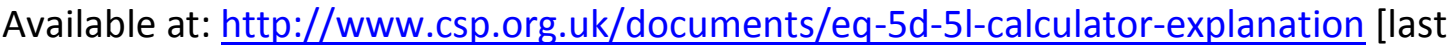
accessed 29th April 2016]

13. Nilsson-Helander K, Thomee R, Gravere-Silbernagel K, Thomee P, Faxen E, Eriksson B, et al. (2007) The Achilles Tendon Total Rupture Score (ATRS): Development and Validation. Am J Sports Med. 2007; 35: 421-426.

14. Kearney RS, Achten J, Lamb SE, Parsons N, Costa ML. The Achilles tendon total rupture score: a study of responsiveness, internal consistency and convergent validity on patients with acute Achilles tendon ruptures. Health and Quality of Life Outcomes. 2012; 10:24.

15. Weber m, Nieman M, Lanz R, Muller T. Non operative treatment of acute rupture of the Achilles tendon. Results of a new protocol and comparison with operative treatment. Am J Sports Med. 2003; 31: 685-91.

16. Sullivan GM, Feinn R. Using Effect Size-or Why the P Value Is Not Enough. J Grad Med Educ. 2012; 4: 279-82

17. Lash L, Horne G, Fielden J, Devane P. Ankle Fractures: Functional And Lifestyle Outcomes At 2 Years. ANZ J Surg. 2002; 72: 724-30

18. Pandis N, Tu YK, Fleming PS, Polychronopoulou A. Randomized and nonrandomized studies: complementary or competing? Am J Orthod Dentofacial Orthop. 2014; 146: 633-40. 
19. Glasziou P, Chalmers I, Rawlins M, McCulloch P. When are randomised trials unnecessary? Picking signal from noise. BMJ. 2007; 334:349-51. 


\section{Tables}

Table 1. Conservative Management of Achilles tendon Rupture

\begin{tabular}{|c|c|c|c|}
\hline Time frame & Bracing \& WB & Physiotherapy & Goals \\
\hline $0-4$ Weeks & $\begin{array}{l}\text { WBAT VACOped } \\
\text { Locked Equinas }\end{array}$ & $\begin{array}{l}\text { Adhoc as required by } \\
\text { patient for boot } \\
\text { comfort. } \\
\text { (To be seen within } 24 \\
\text { hours of patient contact } \\
\text { re concern) }\end{array}$ & \multirow[t]{3}{*}{ Boot comfortable } \\
\hline 4-6 Weeks & $\begin{array}{l}\text { WBAT VACOped } \\
\text { Dynamised 30-20 } \\
\text { plantarflexion }\end{array}$ & \multirow[t]{2}{*}{$\begin{array}{l}\text { Check tendon integrity } \\
\text { with palpation and } \\
\text { resting postion }\end{array}$} & \\
\hline 6-8 Weeks & $\begin{array}{l}\text { WBAT VACOped } \\
\text { Dynamised } 30-10 \\
\text { plantarflexion } \\
\text { Boot off for hygiene, } \\
\text { patient may change } \\
\text { own liner }\end{array}$ & & \\
\hline 8-10 Weeks & $\begin{array}{l}\text { FWB } \\
\text { Boot unlocked 30-0 } \\
\text { Small Sole } \\
\text { Boot off overnight }\end{array}$ & $\begin{array}{l}\text { Sitting heel raises } \\
\text { Resistance band for Pf } \\
\text { con / dfecc }\end{array}$ & $\begin{array}{l}\text { Early strengthening. } \\
\text { Good gait in boot }\end{array}$ \\
\hline 10-15 Weeks & $\begin{array}{l}\text { FWB } \\
\text { Heel raise, reduce by } \\
\text { one layer a week }\end{array}$ & $\begin{array}{l}\text { Progressive } \\
\text { strengthening and } \\
\text { proprioception work }\end{array}$ & Normal walking Gait \\
\hline 16 Weeks + & Normal footwear & $\begin{array}{l}\text { Impact work as } \\
\text { necessary }\end{array}$ & $\begin{array}{l}\text { Goal driven } \\
\text { individuialised } \\
\text { rehabilitation }\end{array}$ \\
\hline
\end{tabular}

WBAT weight bearing as tolerated; FWB full weight bearing 
Table 2. Participant Demographics

\begin{tabular}{|l|l|l|l|}
\hline & Number & Age Mean (SD) & BMI Mean (SD) \\
\hline Male & 13 & $49.1(11.1)$ & $27.6(2.5)$ \\
\hline Female & 8 & $47.6(10.8)$ & $28.1(6.4)$ \\
\hline
\end{tabular}

Figures

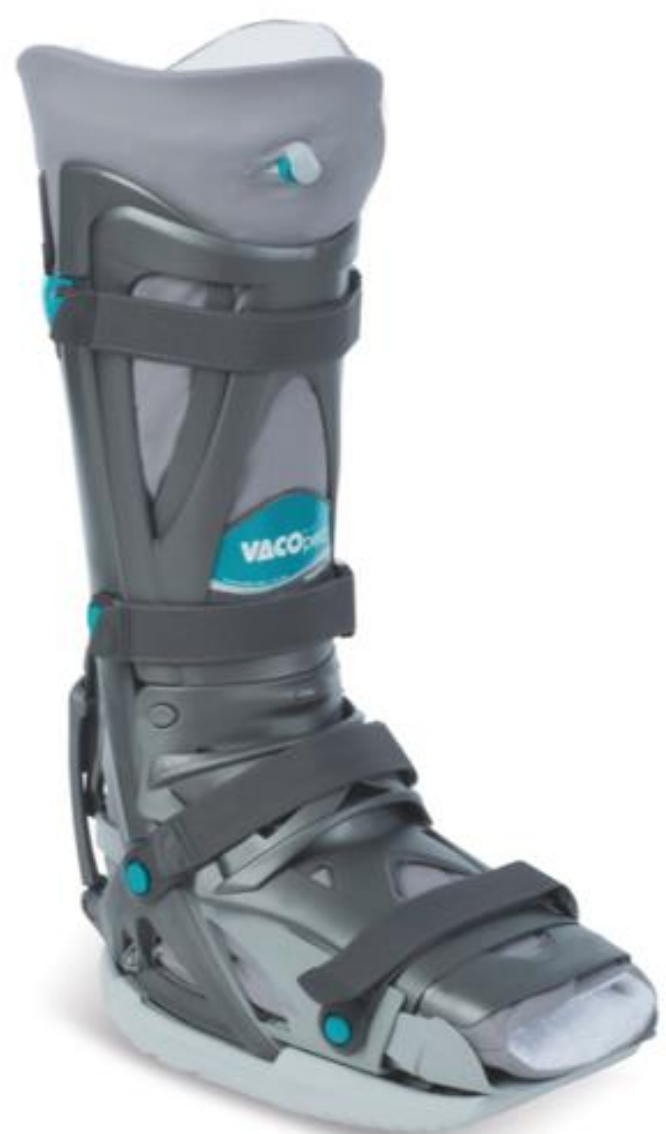

Figure 1. VACOped boot 


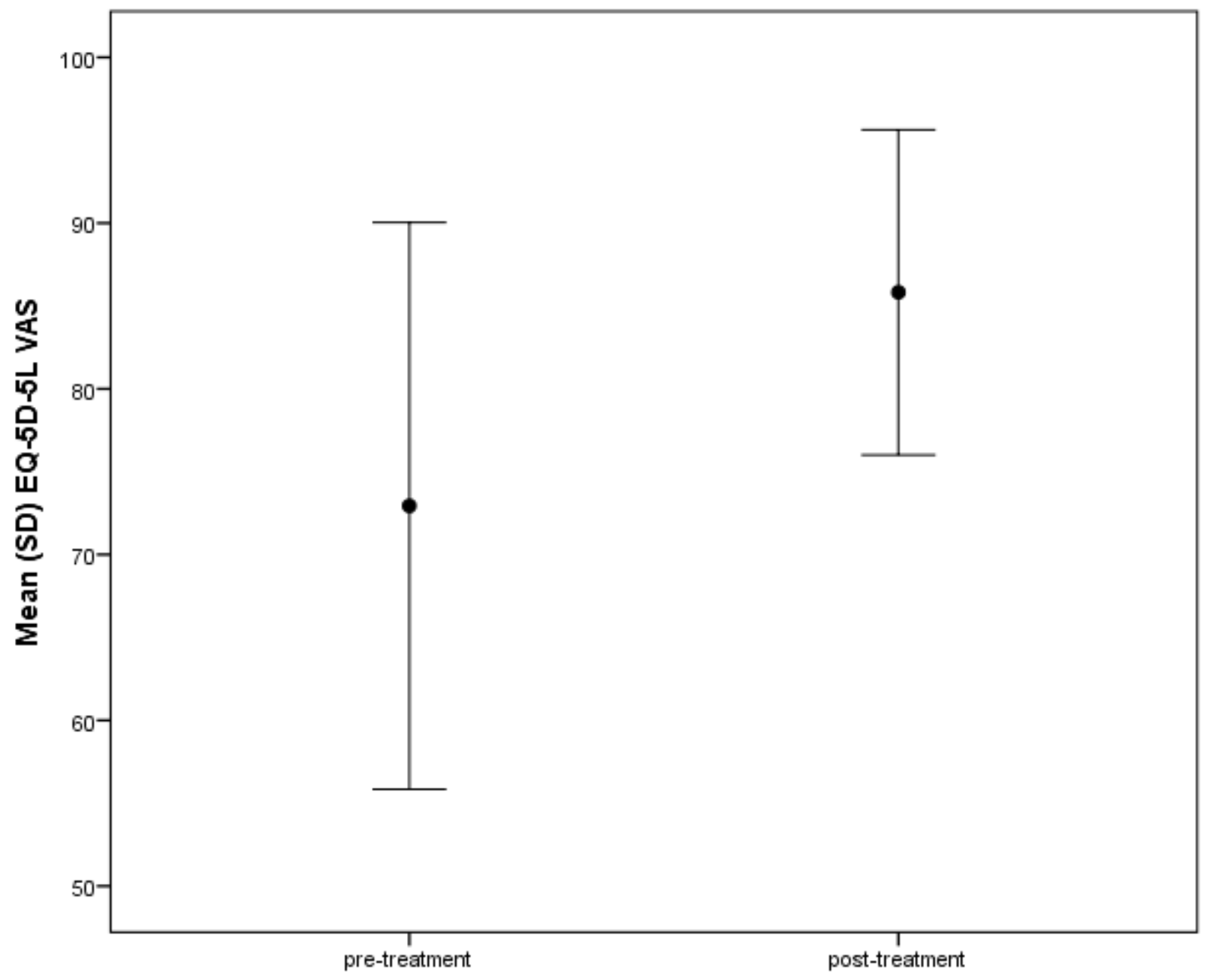

Figure 2. EQ-5D-5L VAS pre- and post-treatment 